\title{
Effect of 5 weeks radiation therapy on pulmonary functions in breast cancer patients: A pilot study
}

\author{
Shastri Nikita $A^{1}$, Diwate Abhijit D², Das Arijit K ${ }^{3}$, Reshma Shete ${ }^{4}$
}

\begin{abstract}
${ }^{1} \mathrm{MPT} 2^{\text {nd }} y r$. Cardiovascular \& Respiratory Sciences PT DVVPF's College of Physiotherapy, Ahmednagar, India ${ }^{2}$ Prof \& HOD, ${ }^{2}$ Associate Professor, ${ }^{2}$ Assistant Professor, Dept. of Cardiovascular \& Respiratory Sciences, PT DVVPF's College of Physiotherapy, Ahmednagar, India
\end{abstract}

\begin{abstract}
Background: The lung has a tiny regenerative capacity; it cannot tolerate prolonged doses of radiation. The critical injuries that eventually lead to impaired ventilation and diffusion capacity are related to the total amount, its fractionation, and the volume of lung irradiated. The absorption of ionizing radiation causes immediate subcellular and cellular damage while its gross morphological expression in terms of tissue injury. Aim: To observe the effect of 5 weeks of Radiation Therapy on Pulmonary Functions in breast cancer patients. Methodology: The study was carried out on ten subjects ranging in the age group of 35-65 years receiving radiation therapy where PFT parameters were recorded initially and at the end of every week for five weeks. Then parameters were compared pre radiation and every week after the radiation therapy for five weeks. Result: Data was analyzed in terms of the mean difference. There were no significant changes that were found in the parameters of the pulmonary function test. Conclusion: Changes in lung volume and capacities are expected after Radiation therapy but patient never present changes due to compensation concerning healthy changes which did not receive radiation. The current study concluded that no significant changes were seen in parameters of Pulmonary Function test that is pre radiation and end of every week for five weeks of Radiation Therapy in breast cancer patients.
\end{abstract}

KEYWORDS: Radiation therapy; Pulmonary function test; Breast cancer

\section{INTRODUCTION}

$\mathrm{B}$ reast cancer is one of the most common malignancies in women in India and affects almost 80,000 women each year [1]. It can be a life-threatening disease for women worldwide. In 2019 an estimated 2,68,000 new cases of invasive breast cancer were diagnosed among women. Over 1,00,000 new breast cancer patients are estimated to be diagnosed annually in India [1]. Approximately 41,760 women died from breast cancer in 2019 [1].

"Breast cancer is a group of diseases in which cells in breast tissue changes and divide uncontrolled, resulting in lump or mass" [1].

Most of the breast cancers are treated with conservatively and surgery, followed by radiation therapy.

Depending on the stage and type of the tumor, lumpectomy (removal of the lump only), or

Correspondence: Dr.Nikita, Cardiovascular \& Respiratory Sciences PT DVVPF's College of Physiotherapy Ahmednagar, Maharashtra, India.. Email: nikitashastri1995@gmail.com

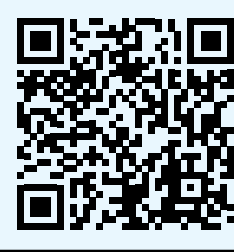


surgical removal of the entire breast (mastectomy) is performed. Standard practice requires the surgeon to establish that the tissue removed in operation has margins clear of cancer, indicating that cancer has been completely excised. If the removed tissue does not have clear margins, further operations to remove more tissue may be necessary. This may sometimes require the removal of part of the pectoralis major muscle, which is the main muscle of the anterior chest wall. More recently, the technique of sentinel lymph node (SLN) dissection has become popular, as it requires the removal of far fewer lymph nodes, resulting in fewer side effects.

Surgery for breast cancer consists of two main options.

1) In breast-conserving surgery, only the tumor and an area of normal tissue surrounding it are removed. Breast-conserving surgery includes the following:

a) Lumpectomy: A small amount of surrounding normal tissue is removed.

b) Quadrantectomy: About one-fourth of the breast is removed.

2) In a mastectomy, all breast tissues are removed [2].

Radiation therapy involves using high-energy $\mathrm{X}$-rays or gamma rays that target a tumor or post-surgery tumor site. These radiations are very effective in killing cancer cells that may remain after surgery or recur where the tumor is removed. In addition to this treatment, implanted radioactive catheters (brachytherapy) involve radiation delivered from a tiny source embedded directly into or next to the tumor. These sources produce gamma-rays, which have the same effect on cancer cells as X-rays. This treatment reduces damage to surrounding healthy tissue, thereby limiting side effects, similar to those used in prostate cancer treatment. However, this treatment option has been superseded by electron beam radiotherapy to the breast scar. Radiation therapy for breast cancer is usually performed after surgery and is an integral component of breast-conserving therapy. The dose of radiation must be healthy enough to ensure the elimination of cancer cells. Treatments are typically given over a period of five to seven weeks, performed five days a week. Each treatment takes about 15 minutes.

If breast-conserving surgery is done, radiation therapy is usually given after surgery to lower the chance of recurrence.

Women who have breast-conserving surgery will be treated with radiation therapy after surgery, and who undergone mastectomy will be treated with radiation if cancer cells are found in lymph nodes. Cancer treated with neo-adjuvant (before surgery) drug like trastuzumab \& pertuzumab is given which shrink the tumor and allow to have breast-conserving surgery if mastectomy is not done. This stage is commonly treated with surgery and radiation therapy [2].

The effect of radiation on the lung leads to physiological changes. However, since the lung as a whole has little regenerative capacity, it cannot tolerate large doses of radiation, and its radiosensitivity is the major limiting factor in radiotherapy of the chest. The effect of irradiation is Radiation-induced pneumonitis usually becomes apparent approximately 2-4 months after irradiation. Clinical symptoms include cough, low-grade fever, dyspnea, fatigue, and pleuritic chest pain, and radiationinduced fibrosis that occurs after six months of radiation, these reduce the functional capacity of lungs. Irradiation causes adverse effects on exercise capacity, respiratory muscle strength, and chest wall mobility. Irradiation of a volume in the apex of the lung cause less functional deficit than a similar amount in the base of the lung. It is well established that the absorption of ionizing radiation causes immediate biochemical, subcellular, and cellular damage, while its morphological expression in terms of gross tissue injury and organ dysfunction is often considerably delayed [3].

A progressive disease, multiple medical interventions leads to mental stress, impairment in a physical condition which includes neuromuscular and skeletal structure dysfunction, functional impairment leads to loss of muscle strength, fatigue, and balance impairment affecting cancer patients. Physical activity and exercise training of breast cancer patients with early stage of the disease and cancer survivors is shown to improve physical functioning, and to reduce fatigue. Distressing symptoms like pain and fatigue with the total number of functional impairment leads to two-third of variation in functional outcomes. Uncomfortable symptoms and an activity limiting symptom (Fatigue) affects lung capacity in the cancer patient. The study aims to observe the effects of 5 weeks of Radiation Therapy on Pulmonary Functions in Breast Cancer Patients [4].

Our aim of the study is to observe short term or acute effects of radiation therapy on lung physiology and lung parenchyma and to find out whether changes take place initially if though, so early intervention can lead to avoiding the changes that are radiation fibrosis which occurs after six months. So this study is undertaken to observe acute short term effects of radiation therapy on lung parenchyma for five weeks. 


\section{Material And Methodology}

Study design: It is an observational study

Ethical approval: Institutional Ethical Committee Approval was taken from the Institutional Ethical Committee, which is approved by the government body.

Study place: The study was conducted in the Tertiary Medical Care Hospital with the attachment of the oncology department.

Inclusion criteria: The patients were included between the age group of 35-65 years female who underwent Modified Radical Mastectomy with neoadjuvant and adjuvant chemotherapy. All patients received external beam radiotherapy with a linear accelerator for five weeks, the technique used was VMAT (volumetric modulated arc therapy) dose was 5040 cGy (50.4 Gy) 28 fraction/180 Gy per fraction.

Exclusion criteria: Patients who diagnosed with Respiratory Disease, history of smoking, any contraindications for spirometry like unstable angina, myocardial infarction, or changes cardiac functions, aorta aneurysm, cerebral aneurysms, and syncope associated with forced exhalation were excluded

Sample size: Ten

\section{Methodology}

According to ATS guidelines, the following procedure was followed:

The patient's age, height, weight were recorded, BMI was calculated. The therapist explained the procedure of Pulmonary Function tests to patients. Proper upright sitting positioning of the patient was done with arm resting by the side of the chair. Patients were instructed to loosen their tight-fitting clothes. Instruct the patient to inhale deeply through the nose; the nose clip was attached. Place the mouthpiece and close the lips around the mouthpiece and exhale maximally until no more air is expelled while maintaining an upright position. After deep expiration, the patient was instructed to inhale by mouth maximally, and PFT parameters that are FEV1, FVC, FEV1/FVC, PEFR, FEF 25-75\% were taken at end of every week for 5 weeks [5].

Statistical analysis: The data collected were analyzed using the SPSS version. Data was compared that is pre-radiation and end of every week for five weeks, and the Friedman test was performed.

\section{RESUlts}

10 patients were taken for the study based on inclusion and exclusion criteria Baseline characteristic showed in table 1
Table1. Baseline characteristic of demographic data of 10 patients

\begin{tabular}{|l|l|}
\hline Demographic data & Mean \\
\hline Age (years) & $52.5 \pm 7.63$ \\
\hline Height $(\mathrm{cm})$ & $15.6 \pm 6.39$ \\
\hline Weight $(\mathrm{kgs})$ & $59.5 \pm 10.49$ \\
\hline
\end{tabular}

Table 2. Comparison of Effects of 5 weeks of Radiation Therapy on Pulmonary function Test in Breast Cancer Patient

\begin{tabular}{|c|c|c|c|c|c|}
\hline \multirow[t]{2}{*}{ RANKS } & \multicolumn{5}{|c|}{ Mean rank } \\
\hline & $\begin{array}{c}\text { FVC } \\
(\mathrm{L})\end{array}$ & $\begin{array}{c}\text { FEV1 } \\
\text { (L) }\end{array}$ & $\begin{array}{c}\text { FEV1/ } \\
\text { FVC (L) }\end{array}$ & $\begin{array}{c}\text { FEF } \\
25-75 \% \\
(\mathrm{~L})\end{array}$ & $\begin{array}{c}\text { PEFR } \\
\text { (L/Min) }\end{array}$ \\
\hline predicted & 4.2 & 4.15 & 3.1 & 5.2 & 7 \\
\hline $\begin{array}{c}\text { Pre } \\
\text { radiation }\end{array}$ & 4.45 & 4.3 & 4.5 & 4 & 4.8 \\
\hline $1^{\text {st }}$ week & 4.2 & 3.55 & 3.7 & 3.7 & 3.8 \\
\hline $2^{\text {nd }}$ week & 4.5 & 3.9 & 4.35 & 3.75 & 3.65 \\
\hline $3^{\text {rd }}$ week & 4.4 & 4.75 & 4.9 & 4.45 & 3 \\
\hline $4^{\text {th }}$ week & 3.5 & 3.85 & 4.1 & 3.85 & 3.2 \\
\hline $5^{\text {th }}$ week & 2.75 & 3.5 & 3.35 & 3.05 & 2.55 \\
\hline \multicolumn{6}{|c|}{ Test Statistics } \\
\hline $\mathrm{N}$ & 10 & 10 & 10 & 10 & 10 \\
\hline $\begin{array}{l}\text { Chi- } \\
\text { Square }\end{array}$ & 5.456 & 2.549 & 5.458 & 5.978 & 29.715 \\
\hline df & 10 & 10 & 10 & 10 & 10 \\
\hline Sig & .487 & .863 & .487 & .426 & .000 \\
\hline
\end{tabular}
$>0.05$ between groups' parameters that are pre-radiation and every week after radiation for five weeks.

\section{Discussion}

The study was conducted on the effect of 5 weeks of radiation therapy on pulmonary functions in breast cancer patients. In this study, all parameters that are before and after radiation therapy changes were observed, and the changes were statistically not significant. But clinically in the case of FEV1 which signifies larger airway obstruction shows increase in the first week when compared with pre-radiation but the sudden reduction in 2 nd week was seen and gradual improvement in 3rd 4th and 5 th week was observed. Some changes were seen in FVC, PEFR value, but in the case of FEF25-75\%, which signifies smaller airway obstruction is not seen in pre and post-radiation. But when considering FEV1/FVC, which is an important parameter to decide whether lung has obstructive/restrictive changes, it shows no many changes that are pre and post-radiation.

Current days as radiation therapy technology has improved a lot. Pinpointed radiation and little exposure to radiotherapy for lung reduce lung damage drastically. As this institute, one of the 
latest technology that is volumetric modulated arc therapy (VMAT). So it will hardly damage the lung. But clinical changes still show because of surgery, adjuvant chemotherapy, which is very little changes whether they are reversible or not to know that long term study will be required.

Before starting radiation, we planned in such a way that tolerance of lungs should not get exceeded (V20<20 Gy), which is $20 \%$ volume of the lung should not receive more than $20 \mathrm{~Gy}$.

Dayane Evelyn et al. has done a study on the effect of radiation therapy on pulmonary function and fatigue of women undergoing treatment for breast cancer. He said that there was a reduction in pulmonary function measurement was there despite this value remain within normality according to weight height age of the sample.

Changes in lung capacity and volume are expected after radiation therapy; therefore, the risk of damaging pulmonary parenchyma, type 2 pneumocystis, however, there is a possibility of patients never show any changes due to compensation with healthy lung which do not receive radiation.

In our study, no significant changes were seen in pulmonary function parameters within the week and after five weeks of radiation therapy [6].

A study by Eyad Fawzi et al. concluded that reduction of FVC, FEV1 was seen with ipsilateral lung V20 at day 90 after post-mastectomy radiation therapy.

Reduction of FEV1 and FVC with standard FEV1\% pre suggest an acute exudative inflammatory process in the alveolar space, favoring restrictive lung injury pattern [7].

Anya Jafari et al. did a study on Pulmonary Function Test changes two months after breast Radiotherapy; they concluded that no significant correlation between volumes of irradiated lung and spirometry parameters, so with increased volume of the lung there was no decrease in spirometry parameters (FEV1, FVC, FEV1/ FVC). The radiotherapy of the breast is causing impaired pulmonary function tests. However, considering healthy tissue tolerance doses during planning, we would expect lower lung complications [8].

Despina Spyropoulou et al. conducted study on Pulmonary Function Testing in Women with Breast Cancer Treated with Radiotherapy and Chemotherapy and concluded that there was a significant decrease of forced vital capacity, forced expiratory volume in one second and $\mathrm{CO}$ diffusing capacity was observed in the women treated with locoregional adjuvant radiotherapy and chemotherapy. In contrast, to decrease of the above parameters was evidenced in women treated exclusively with local adjuvant radiotherapy.

Concluded that Local adjuvant radiotherapy is not associated with any reduction in lung function parameters; however, locoregional adjuvant radiotherapy combined with chemotherapy shows a significant decrease in PFTs 3 months after radiotherapy completion.

The decrease of FVC and FEV1, but the stable relationship between them may also indicate restrictive lung injury explained by the fact that changes in FVC and FEV1 may reflect an acute exudative process in the alveolar space.

VO2max is an indicator of the physical fitness of a person, and the fact that it remained unchanged indicated that although FVC, FEV1, and DLCO decreased, this result did not reflect any clinical symptoms most probably because the healthy lung compensated for the radiation lung injury [9].

Mathew Q.F.Hatton, Desni Nixon et al. conducted a study on acute change in expiratory flow rate following palliative radiotherapy for bronchial carcinoma; they found that there were pathophysiological changes that were seen within few hours of irradiation, with changes within leading to release of mediators which can initiate inflammatory reactions. This can lead to radiation pneumonitis and fibrosis, though short term, the inflammation may exacerbate the bronchial obstruction already present. The small changes in patency of significant airways may lead to a massive difference in respiratory function tests.

Therefore a patient with malignancy affects extensive central airway experiences that fall in peak expiratory flow rate in hours after treatment [10].

\section{Conclusion}

The study concluded that no significant changes were seen in parameters of Pulmonary Function test that is pre radiation and end of every week for five weeks of Radiation Therapy in breast cancer patients.

Future scope: With more samples, the same study may continue to get a reliable result. To avoid further damage to lung parenchyma that leads to radiation fibrosis, we can initiate early intervention to patients like lung expansion exercise, which will increase the expansion capacity of lungs, and the restrictive pattern will be avoided and due to early intervention lung patency will be maintained.

Conflict of interest: No conflict of interest. 
Acknowledgment: I am thankful to Dr. Deepak Anap, Dr. Archana Nagargoje, for helping me in the study and grateful to my colleagues.

\section{REFERENCES}

1. American Cancer Society. Breast Cancer Facts \& Figures 2019-2020. Atlanta: American Cancer Society, Inc. 2019.

2. Sharma GN, Dave R, Sanadya J, Sharma P, Sharma KK. Various types and management of breast cancer: an overview. Journal of advanced pharmaceutical technology \& research. 2010;1(2):109

3. Coggle JE, Lambert BE, Moores SR. Radiation effects in the lung. Environmental health perspectives. 1986;70:261-91

4. Kokkonen K, Saarto T, Mäkinen T, Pohjola L, Kautio H, Järvenpää S, PuustjärviSunabacka K. The functional capacity and quality of life of women with advanced breast cancer. Breast Cancer. 2017;24(1):128-36

5. Brusasco V, R.Crapo. ATS/ERS Stnadardisation of Lung Function Testing. Eur Respir J. 2005;26:153-61
6. Santos DE, Rett MT, Mendonça AC, Bezerra TS, Santana JM, Silva Júnior WM. Effect of radiotherapy on pulmonary function and fatigue of women undergoing treatment for breast cancer. Fisioterapia e Pesquisa. 2013;20(1):50-5

7. Al Saeed EF, Balaraj FK, Tunio MA. Changes in pulmonary function tests in breast carcinoma patients treated with locoregional post-mastectomy radiotherapy: results of a pilot study. Breast cancer. 2017;9:375-81

8. Jafari, Anya Malekzadeh, Mona. Pulmonary function test changes two months after breast radiotherapy - 2 - Asian Pacific Journal of Cancer Care. 2019;2(2):21-3

9. Spyropoulou D, Leotsinidis M, Tsiamita M, Spiropoulos K, Kardamakis D. Pulmonary function testing in women with breast cancer treated with radiotherapy and chemotherapy. in vivo. 2009; 23(5):867-71.

10. Matthew Q.F Hatton. Acute changes in peak expiratory flow rate following palliative Radiotherapy for bronchial carcinoma. Elsevier Inc. 1997:44(1):31-4 